\title{
Validación y ajuste del modelo hidrogeológico conceptual de un humedal de la Llanura Pampeana, Provincia de Buenos Aires, Argentina
}

\author{
Asunción Romanelli ${ }^{1,2, *}$, Orlando Mauricio Quiroz Londoño ${ }^{1}$, Hector Enrique Massone ${ }^{1}$ y Ali- \\ cia Haydée Escalante ${ }^{2,3}$
}

${ }^{1}$ Universidad Nacional de Mar del Plata. Facultad de Ciencias Exactas y Naturales, Instituto de Geología de Costas y del Cuaternario. Funes 3350. Piso 1. (7600 Mar del Plata, Argentina).

${ }^{2}$ Consejo Nacional de Investigaciones Científicas y Técnicas (CONICET).

${ }^{3}$ Universidad Nacional de Mar del Plata. Facultad de Ciencias Exactas y Naturales, Departamento de Biología, Laboratorio de Limnología. Funes 3250. Piso 2. (7600 Mar del Plata, Argentina).

*Autor responsable de la correspondencia: asuncionromanelli@hotmail.com; aromanel@mdp.edu.ar

Recibido: 6/4/10

Aceptado: $21 / 7 / 10$

\begin{abstract}
Validation and adjustment of the conceptual hydrogeological model for a wetland of the Pampa Plain, Buenos Aires Province, Argentina

Understanding the hydrological functioning and the interaction among the different water bodies present in a specific area are essential when planning the rational use of the hydric resource. The aim of the present paper is to validate and adjust the conceptual hydrogeological model for a wetland of the Buenos Aires Pampa Plain through hydrochemical and isotopic techniques. La Brava Wetland Basin $\left(53.6 \mathrm{~km}^{2}\right)$ has been selected. La Brava is a shallow and permanent wetland with an area of $4.0 \mathrm{~km}^{2}$ and a maximum depth of $4.57 \mathrm{~m}$. Samples for physicochemical and isotopic analyses were taken from groundwater, the wetland, and in the inflow and outflow streams. A regular grid was designed for the wetland sampling to measure in situ the main physical and chemical parameters at 5 different depths. The chemical composition of the water in the basin is of sodium bicarbonate type. Hydrochemical and isotopic data allowed the confirmation of the effluent-influent behavior of this wetland. Electrical conductivity analysis in the wetland at different depths shows a possible preferential recharge zone in the contact of the wetland with La Brava Hill, where an important decrease in this parameter was detected. The information obtained indicates that the recharge to the aquifer is influenced by: the rainfall, the wetland discharge, the concentration of surface run-off from the range fringes to inter-range depressions and water input from the fault system present in the quartzites.
\end{abstract}

Key words: Hydrogeological model, Hydrochemistry, Stable isotopes, Wetland.

\section{RESUMEN}

Validación y ajuste del modelo hidrogeológico conceptual de un humedal de la Llanura Pampeana, Provincia de Buenos Aires, Argentina

Comprender el funcionamiento hidrológico y la interacción de los diferentes cuerpos de agua presentes en un área específica son temas esenciales a la hora de planificar el uso racional del recurso hídrico. El objetivo de este trabajo es validar y ajustar el modelo hidrogeológico conceptual de un humedal de la llanura Pampeana Bonaerense mediante técnicas hidrogeoquímicas e isotópicas. Se ha seleccionado la cuenca de la laguna La Brava $\left(53.6 \mathrm{~km}^{2}\right)$. La misma es una laguna permanente y somera con una superficie de $4.0 \mathrm{~km}^{2}$ y una profundidad máxima de $4.57 \mathrm{~m}$. Se tomaron muestras para análisis fisicoquímico e isotópico en el agua subterránea, en la laguna y en los arroyos afluente y efluente a la misma. Para el muestreo en la laguna se diseñó una malla de muestreo regular en la cual se midieron in situ los principales parámetros fisicoquímicos a 5 profundidades diferentes y se tomaron muestras. La composición química del agua de la cuenca es de tipo bicarbonatada sódica. El carácter efluente-influente de este humedal pudo ser confirmado a través de las mediciones isotópicas e hidroquímicas. El análisis de la conductividad eléctrica en el humedal a diferentes profundidades hace pensar en una zona de recarga preferencial en el contacto de la laguna con el faldeo oriental de la Sierra La Brava, donde se evidencia una disminución 
importante de este parámetro. La información obtenida indica que la recarga al acuífero en esta zona es influenciada por: la precipitación, la descarga proveniente del humedal, la concentración del escurrimiento superficial desde el frente montañoso hacia las depresiones interserranas y el aporte de agua proveniente del sistema de fisuras presente en las ortocuarcitas.

Palabras clave: Modelo hidrogeológico, Hidroquímica, Isótopos estables, Humedal.

\section{INTRODUCCIÓN}

Los cuerpos de agua superficial son parte activa del sistema de flujo del agua subterránea (Winter, 1999). En diferentes estudios se han considerado las condiciones de flujo subterráneo y sus relaciones con el flujo superficial, destacando la importancia ecológica de estas relaciones, los impactos humanos y su influencia con la disponibilidad del recurso (Sophocleus, 2002). Elaborar modelos conceptuales que expliquen adecuadamente el funcionamiento y relación de los diferentes cuerpos de agua en una zona, resulta esencial a la hora de generar políticas de gestión que favorezcan el adecuado aprovechamiento del recurso hídrico.

En el sudeste de la provincia de Buenos Aires las aguas subterráneas constituyen la principal fuente de abastecimiento para fines urbanos, agrícolas e industriales. Los ríos y arroyos, si bien no son significativos desde el punto de vista de abastecimiento, lo son desde la dinámica de los procesos hidrológicos. Por su parte, los humedales, numerosos en esta zona, proporcionan importantes servicios ambientales como el ciclado de nutrientes, la regulación del clima a nivel local y regional, regulación de flujos hidrológicos, soporte ecosistémico esencial y oportunidades educacionales y recreativas entre otros (MA, 2005). Pese a la importancia económica y ambiental de estos cuerpos de agua, en la mayoría de los casos se carece de información que permita definir de manera precisa un modelo conceptual de funcionamiento.

Una herramienta importante aplicada al análisis de sistemas hidrológicos es la interpretación hidrogeoquímica, la cual permite determinar distintos tipos de agua, interacción y mezcla entre ellos y posibles procesos modificadores a lo largo de líneas de flujo mediante el análisis del conteni- do y variación de las especies iónicas (Martínez et al., 2000). De igual manera, la aplicación de técnicas isotópicas en los estudios hidrogeológicos ha demostrado ser otra importante herramienta en la validación y ajuste de modelos conceptuales (Clark \& Fritz, 1997; Mazor, 1991; Fontes, 1980). Los procesos físicos y los fenómenos meteorológicos responsables del transporte del agua en las diferentes fases del ciclo hidrológico producen un fraccionamiento isotópico que puede ser aprovechado para obtener conclusiones sobre su origen y comportamiento. Esto permite definir, entre otros aspectos, las relaciones entre las aguas superficiales y subterráneas y zonas de recargadescarga. Estas técnicas han sido implementadas para confirmar y mejorar diversos modelos hidrogeológicos que sirven como herramientas efectivas en el aprovechamiento sustentable del recurso.

El objetivo de este trabajo es validar y ajustar el modelo hidrogeológico conceptual de un humedal de la llanura Pampeana Bonaerense, la Laguna La Brava, mediante técnicas hidrogeoquímicas e isotópicas. Mejorar el conocimiento de las relaciones entre este humedal, el acuífero y los arroyos de la zona, estableciendo sus interacciones y características, es clave para ajustar y/o generar políticas de gestión que ayuden a preservar el recurso hídrico tanto para usos recreativos como hábitat de especies animales y vegetales de manera integral.

\section{CARACTERÍSTICAS DEL ÁREA DE ESTUDIO}

El área de estudio seleccionada comprende a la Cuenca de la Laguna La Brava $\left(53.6 \mathrm{~km}^{2}\right)$, situada sobre el Sistema Serrano de Tandilia (Fig. 1) y perteneciente al Partido de Balcarce al SE de la 


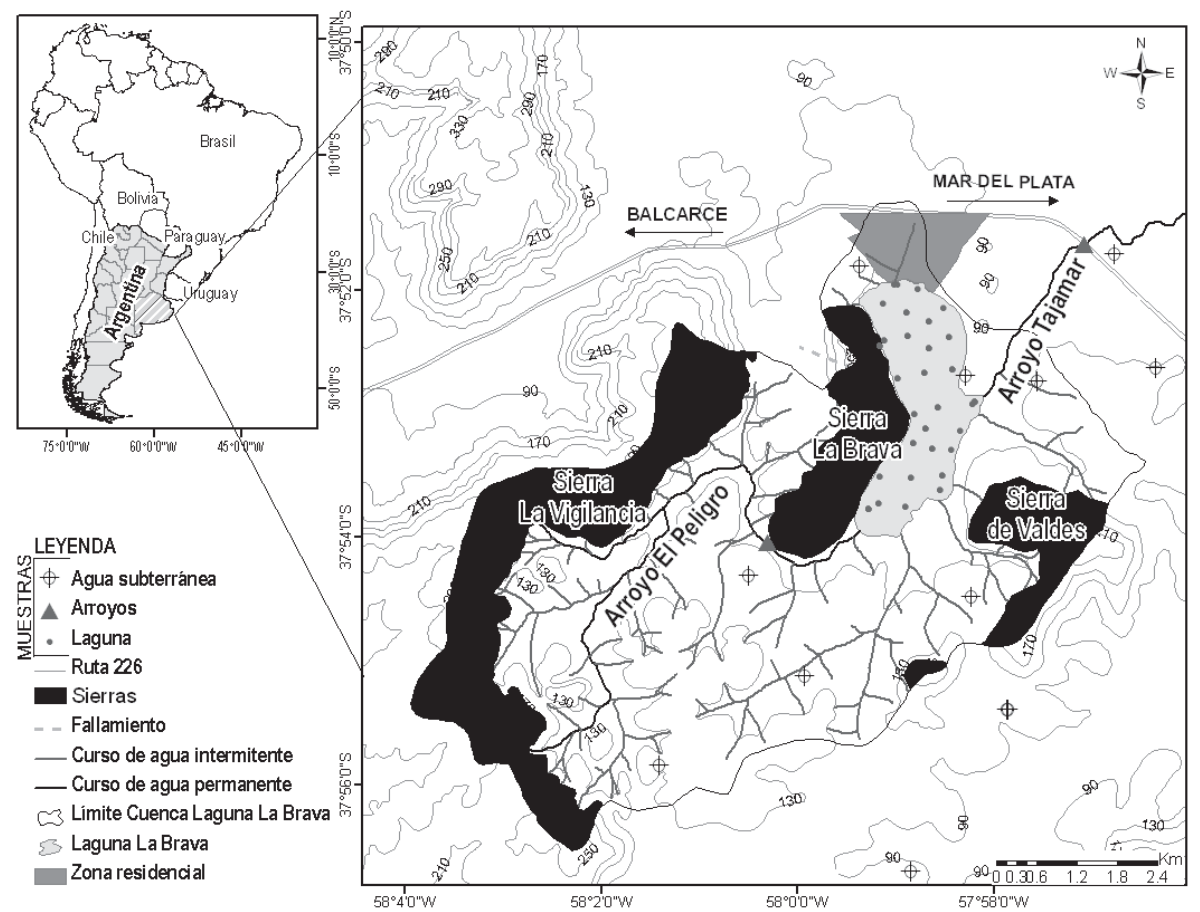

Figura 1. Localización de la Cuenca del humedal La Brava y puntos de muestreo. Location of La Brava wetland watershed and sampling sites.

Provincia de Buenos Aires, Argentina. El clima de la región se clasifica como subhúmedo-seco con nulo o pequeño exceso de agua a los efectos agronómicos (Thornthwaite, 1948). La precipitación promedio para el período 1985-2008 fue de $908.7 \mathrm{~mm} / \mathrm{año}$. El valor promedio de evapotranspiración potencial calculado para 19852007, aplicando el método de Thornthwaite, fue de $732 \mathrm{~mm} / a$ ño. La temperatura media anual para estos años fue de $14.1^{\circ} \mathrm{C}$, con un rango de variación de 7.6 a $20.9^{\circ} \mathrm{C}$. Los rangos de alturas en la cuenca varían entre 65 y 300 msnm. La Laguna La Brava es un cuerpo de agua de régimen permanente, con una superficie de $4.0 \mathrm{~km}^{2}$ (Grosman y Sanzano, 1999) y una profundidad máxima de $4.57 \mathrm{~m}$ (Lirio et al., 2007). El pelo de agua de la misma se encuentra a una cota de $68.03 \mathrm{msnm}$.

El modelo hidrogeológico planteado por Kruse (1987) para este humedal establece una relación efluente-influente con el acuífero. De acuerdo a éste, el humedal recibe un importante aporte subterráneo de las zonas topográficamente más altas, y a su vez, drena subterráneamente un vo- lumen significativo aguas abajo. Superficialmente el mismo recibe un único afluente, el Arroyo El Peligro (SEP), formado por la conjunción de tributarios en el sector alto de la cuenca. Los excesos de agua en la laguna que no pueden ser desalojados subterráneamente, son evacuados a través del Arroyo Tajamar (STJ). SEP es de carácter permanente, mientras que STJ presenta caudal escaso a nulo en épocas secas. La recarga de este humedal se debe esencialmente al aporte del acuífero y del SEP. La distribución de las isopiezas para la zona determina la existencia de un flujo regional con dirección SO-NE (Romanelli et al., 2009).

En la zona de estudio se distinguen dos unidades hidrogeológicas diferentes. La primera, asociada a los sistemas serranos, está compuesta por rocas cristalinas Precámbricas (gneiss, granitos y migmatitas) y una secuencia silicoclástica del Paleozoico inferior (arenitas y sabulitas cuarzosas) que conforman el basamento hidrogeológico del área. Están caracterizadas como un medio discontinuo, anisótropo y heterogéneo con agua en fisuras y productividad de nula a baja. La segun- 
da unidad está constituida por una cubierta cenozoica (sedimentos Postpampeanos y Pampeanos) que conforman el único acuífero del área, denominado "acuífero pampeano". Éste, somero y de tipo libre está constituido esencialmente por intercalaciones de limos y arenas finas de origen eólico y fluvial. Se apoya directamente sobre el basamento hidrogeológico. Es considerado en estudios regionales como "pobre acuífero", asignándoles permeabilidades de hasta $0.5 \mathrm{~m} /$ día llegando localmente a 5 ó $10 \mathrm{~m} /$ día (Sala, 1975).

El límite Oeste de la laguna es coincidente con la Sierra La Brava. Mauriño et al. (1981) definen las características estructurales de esta sierra, estableciendo un escalonamiento de fallas de alto ángulo con diferente rango de inclinación al sureste (Fig. 1) y dos grupos de diaclasas que imprimen una porosidad secundaria importante. La presencia de manantiales intermitentes en esta sierra permite inferir la circulación de agua a través de un sistema de fisuras dentro de las cuarcitas aflorantes en la misma (Tapia, 1937; Kruse, 1987).

Este humedal, localizado en un área netamente agrícola, es considerado un importante centro de recreación y constituye un área útil para la divulgación y educación de la naturaleza. En los últimos años se ha desarrollado en su sector Norte una zona residencial que experimenta un marcado incremento poblacional (300 habitantes sin servicio de agua corriente ni cloacas), con su consecuente presión sobre el sistema. Además, recibe una constante afluencia de visitantes especialmente de las ciudades cercanas, Mar del Plata y Balcarce. Al igual que el resto de los humedales de la llanura pampeana, esta laguna cumple un papel fundamental en la conservación de la biodiversidad proporcionando hábitat a especies residentes y transitorias, permitiendo su supervivencia, alimentación y reproducción (MA, 2005; De Groot et al., 2006).

\section{METODOLOGÍA}

La primera fase de este trabajo consistió en la compilación de datos e información preexistente (topografía, geología, hidrogeología e imágenes satelitales) relacionada con el recurso hídrico.
Se colectaron muestras en la laguna para su análisis hidroquímico y para la determinación de isótopos estables $\left({ }^{18} \mathrm{O}\right.$ y $\left.{ }^{2} \mathrm{H}\right)$, definiendo una malla de muestreo regular de 27 puntos en ella. En cada punto se midieron in situ temperatura, $\mathrm{pH}$ y conductividad eléctrica absoluta (CE) a cuatro profundidades: subsuperficial $(0.5 \mathrm{~m}), 1 \mathrm{~m}$, $2 \mathrm{~m}$ y $4 \mathrm{~m}$, usando una sonda multiparamétrica HANNA HI9828. La precisión de este equipo es $\pm 0.15^{\circ} \mathrm{C}$ para temperatura, \pm 0.02 para $\mathrm{pH}$ y $\pm 1.0 \mu \mathrm{S} / \mathrm{cm}$ en CE. Se seleccionaron 9 puntos en los cuales se colectaron muestras en superficie y a la máxima profundidad para su análisis químico e isótopico, utilizando para el muestreo en profundidad una botella de tipo Van Dorn. También se muestrearon los principales arroyos y 13 captaciones de agua subterránea por bombeo directo, incluyendo pozos de riego, de abastecimiento domiciliario y molinos que captan el acuífero pampeano. Todos los puntos de muestreo se localizaron por medio de un GPS Garmin eTrex Vista.

A todas las muestras de agua se les determinó en laboratorio: iones dinámicos $\left(\mathrm{Ca}^{+2}\right.$, $\mathrm{HCO}_{3}^{-}, \mathrm{CO}_{3}^{-2}$ y $\left.\mathrm{SO}_{4}^{-2}\right)$ y conservativos $\left(\mathrm{Na}^{+}, \mathrm{K}^{+}\right.$, $\mathrm{Mg}^{+2}$ y $\mathrm{Cl}^{-}$). Los métodos empleados fueron: Mohr para los cloruros, turbidimetría para los sulfatos, titulación complejométrica con EDTA para calcio y magnesio, espectrometría de llama para sodio y potasio y titulación potenciométrica para bicarbonatos-carbonatos. Las determinaciones químicas se hicieron siguiendo la metodología detallada en APHA (1992). La información hidroquímica fue analizada mediante una caracterización estadística general y diagramas convencionales de Piper (Hem, 1992), utilizando el programa AQUACHEM 4.0 (Calmbach \& Waterloo Hydrogeologic Inc., 2003). Además, se elaboraron mapas de isovalores usando el software SIG ArcGis 9.2 (ESRI, 2007).

Para el análisis isotópico se recolectaron y analizaron 9 muestras de agua subterránea, 1 muestra de SEP, 1 muestra de STJ y 17 muestras de agua de la laguna La Brava ( 9 superficiales y 8 en profundidad, tomadas a 1 y $3 \mathrm{~m}$ de profundidad, respectivamente, Fig. 2). Se utilizaron para este trabajo los datos isotópicos y contenido de $\mathrm{Cl}$ de la precipitación media mensual obtenidos en la localidad de San Manuel, ubicada $50 \mathrm{~km}$ al 


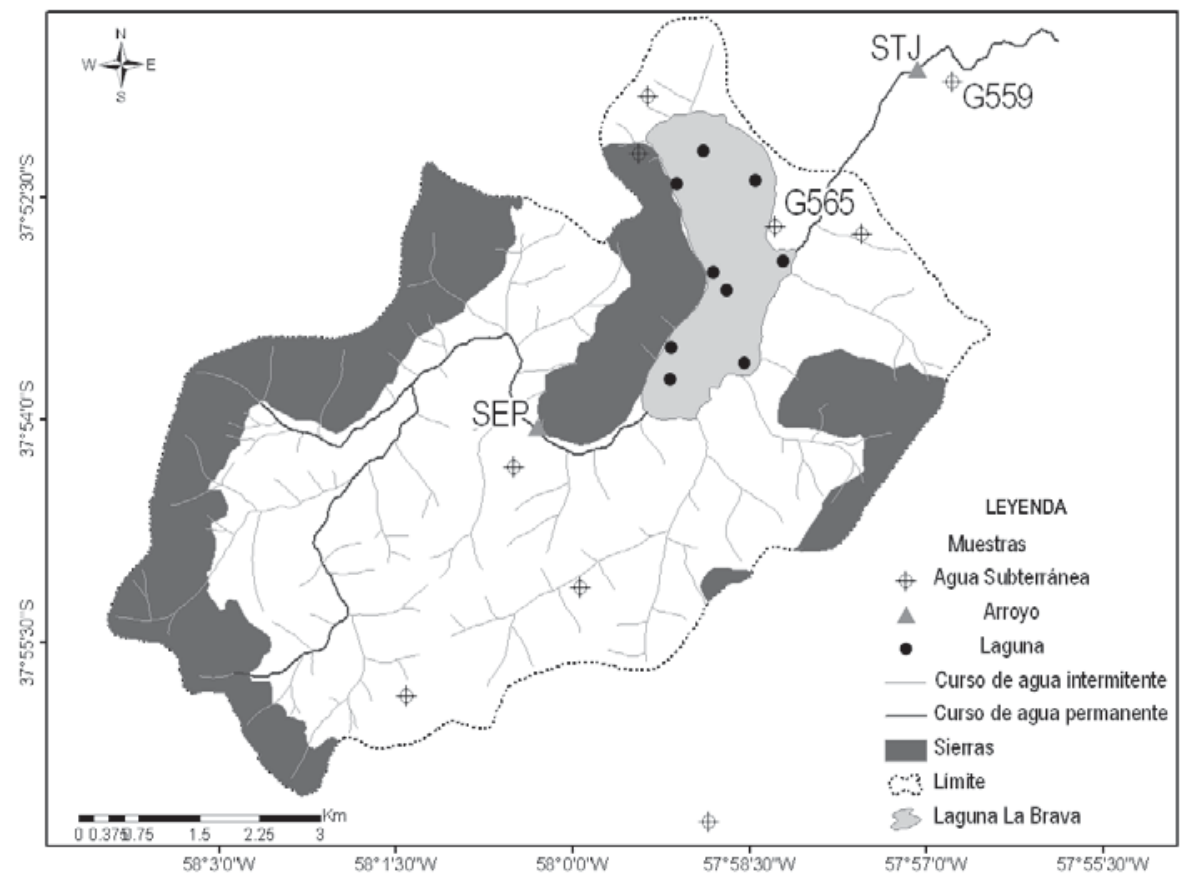

Figura 2. Puntos de muestreo de isótopos $\left(\delta^{18} \mathrm{O}\right.$ y $\left.\delta^{2} \mathrm{H}\right)$. Isotopic sampling sites $\left(\delta^{18} \mathrm{O}\right.$ y $\left.\delta^{2} H\right)$.

Oeste de esta laguna, correspondientes al período comprendido entre el mes de noviembre de 2004 y marzo de 2008 (Quiroz Londoño et al., 2008).

Las determinaciones isotópicas se realizaron mediante un espectrómetro láser DLT-100 Liquid-Water Isotope Analyzer, Automated Injection desarrollado por Los Gatos Research. Los resultados se expresan como $\delta$, definido como: $\delta=1000(R s-R p) / R p \%$, donde, $\delta$ : desviación isotópica en $\%$; $S$ : muestra; $P$ : referencia internacional; $R$ : relación isotópica $\left({ }^{2} \mathrm{H} /{ }^{1} \mathrm{H},{ }^{18} \mathrm{O} /{ }^{16} \mathrm{O}\right)$. La referencia utilizada es Vienna Standard Mean Ocean Water (V-SMOW) (Gonfiantini, 1978). Las incertidumbres son $\pm 0.3 \%$ para $\delta^{18} \mathrm{O}$ y $\pm 1.0 \%$ para $\delta^{2} \mathrm{H}$.

Debido a la ausencia de datos de caudales de los arroyos, se realizó un estudio de mezcla de aguas a partir del ión cloruro (Custodio y Llamas, 1976) para cuantificar la importancia relativa de las principales fuentes de recarga al humedal. Se utilizó este ión por sus características conservativas y la ausencia de fuentes externas del mismo en la cuenca.

Se realizaron mediciones de la profundidad en 27 puntos dentro de la laguna, utilizando una mira topográfica graduada al centímetro. Con esta información, la topografía de la zona y el uso de imágenes de satélite (Landsat 7) se calculó el volumen de agua del humedal para 2008 mediante un proceso preestablecido en el software SIG que calcula áreas y volúmenes entre dos superficies.

El muestreo en la laguna se realizó en un solo día, en el mes de noviembre 2008 (primavera), entre las 9:00 AM y las 18:00 PM. Este mismo día se colectaron muestras tanto de los arroyos como de agua subterránea. Para el caso de los arroyos se cuenta con datos hidroquímicos en diferentes fechas del mismo año. Los análisis fueron realizados en el laboratorio de Hidrogeoquímica e Hidrología isotópica del Instituto de Geología de Costas y del Cuaternario (Universidad Nacional de Mar del Plata).

\section{RESULTADOS}

El mapa batimétrico de la laguna La Brava se muestra en la figura 6.D. El volumen calculado para la fecha de la toma de las mediciones fue de $8.24 \mathrm{hm}^{3}$, y la profundidad máxima registrada fue de $4.10 \mathrm{~m}$. 
Tabla 1. Variabilidad de la composición iónica mayoritaria en el agua de la cuenca del humedal La Brava. Major ionic variability in the water composition of the La Brava wetland wathershed.

\begin{tabular}{lrrrrrrrrrrrrrrrrr}
\hline & \multicolumn{1}{c}{ AGUA SUBTERRÁNEA } & \multicolumn{4}{c}{ LAGUNA } & \multicolumn{4}{c}{ AFLUENTE } & \multicolumn{3}{c}{ EFLUENTE } \\
\hline Parámetro & Mín & Máx & Media & DE & Mín & Máx & Media & DE & Mín & Máx & Media & DE & Mín & Máx & Media & DE \\
\hline $\mathrm{Ca}^{+2}(\mathrm{mg} / \mathrm{l})$ & 5.2 & 34.3 & 15.9 & 8.4 & 19.6 & 55.7 & 31.8 & 8.5 & 28.8 & 52.2 & 40.1 & 10.0 & 14.0 & 39.0 & 21.4 & 10.2 \\
$\mathrm{Mg}^{+2}(\mathrm{mg} / \mathrm{l})$ & 7.2 & 64.4 & 35.5 & 16.3 & 9.0 & 63.1 & 29.5 & 12.8 & 21.4 & 43.7 & 30.3 & 9.5 & 20.0 & 37.8 & 27.4 & 6.5 \\
$\mathrm{Na}^{+}(\mathrm{mg} / \mathrm{l})$ & 105.0 & 398.0 & 249.6 & 84.3 & 107.0 & 271.0 & 194.5 & 53.6 & 126.0 & 266.4 & 183.6 & 61.3 & 111.0 & 306.0 & 170.0 & 73.4 \\
$\mathrm{~K}^{+}(\mathrm{mg} / \mathrm{l})$ & 2.2 & 11.0 & 4.9 & 2.7 & 2.9 & 10.9 & 6.6 & 1.7 & 3.2 & 4.9 & 4.1 & 0.7 & 7.7 & 9.7 & 8.9 & 0.9 \\
$\mathrm{Cl}^{-}(\mathrm{mg} / \mathrm{l})$ & 48.1 & 173.0 & 99.1 & 37.3 & 56.5 & 128.8 & 89.2 & 18.9 & 62.8 & 250.0 & 141.0 & 79.6 & 57.0 & 173.0 & 89.3 & 42.8 \\
$\mathrm{HCO}_{3}^{-}(\mathrm{mg} / \mathrm{l})$ & 240.0 & 894.2 & 484.2 & 164.4 & 224.0 & 857.5 & 418.4 & 179.6 & 282.0 & 388.7 & 334.3 & 44.8 & 320.0 & 1041.2 & 559.0 & 263.1 \\
$\mathrm{CO}_{3}^{-2}(\mathrm{mg} / \mathrm{l})$ & 0.0 & 53.3 & 32.8 & 24.0 & 42.6 & 171.5 & 84.3 & 41.9 & 0.0 & 0.0 & 0.0 & 0.0 & 0.0 & 63.9 & 10.7 & 26.1 \\
$\mathrm{SO}_{4}^{-2}(\mathrm{mg} / \mathrm{l})$ & 1.0 & 48.3 & 17.4 & 15.1 & 2.2 & 77.6 & 26.9 & 15.7 & 17.8 & 66.4 & 31.9 & 23.2 & 4.9 & 76.8 & 27.9 & 25.9 \\
\hline
\end{tabular}

\section{Caracterización Hidroquímica}

La Tabla 1 muestra los principales parámetros estadísticos del contenido iónico de los distintos tipos de aguas en la Cuenca de la Laguna La Brava.

\section{Agua subterránea}

La variación de facies hidroquímicas fue analizada mediante un diagrama triangular de Piper (Fig. 3 A). El agua subterránea en la cuenca es

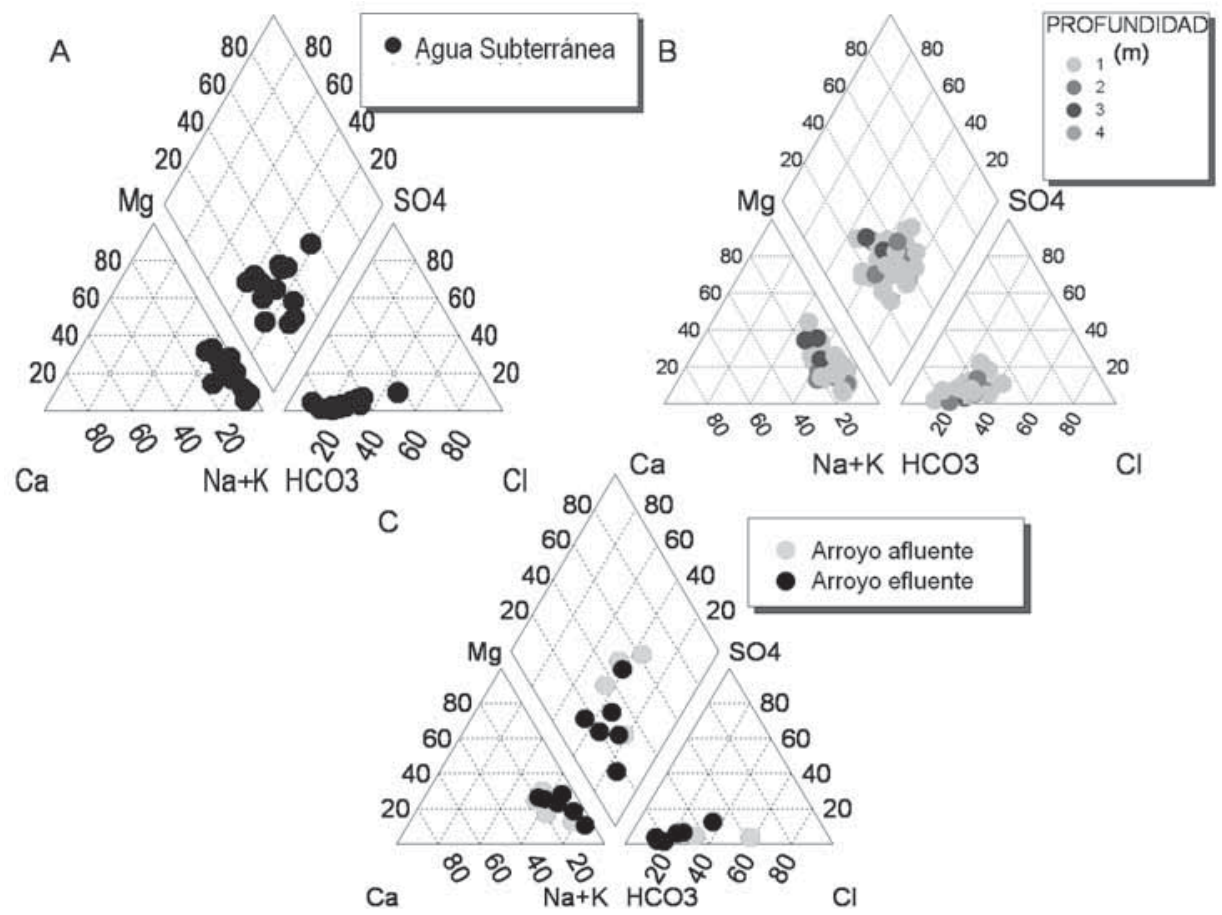

Figura 3. Contenido iónico de A) agua subterránea, B) humedal La Brava (a distintas profundidades) y C) arroyos. Ionic content of A) groundwater, B) La Brava wetland (at different depths), and C) streams. 
de tipo bicarbonatada sódica, presentando gran homogeneidad en su composición catiónica. En su contenido aniónico dominan los bicarbonatos, existiendo algunas muestras con mayor contenido en cloruros (140 mg/l a $173 \mathrm{mg} / \mathrm{l})$ localizadas en la zona norte. Se determinaron valores medios de $\mathrm{CE}$ de $830 \mu \mathrm{S} / \mathrm{cm}$, de $\mathrm{pH} 7.9$ y temperatura de $16.5^{\circ} \mathrm{C}$. Según estos parámetros las aguas subterráneas son levemente alcalinas y de moderada salinidad.

$\mathrm{Al}$ analizar la distribución espacial de la CE en el área se observó en general un aumento regional en dirección SO-NE. Las zonas con CE más bajas $(<800 \mu \mathrm{S} / \mathrm{cm})$ se localizan al Sur y Suroeste de la cuenca coincidentes con el sector topográficamente más elevado, asociadas a las zonas de recarga evidenciadas con la piezometría propuesta por Kruse (1987) y Romanelli et al. 2009 (Fig. 4). Las CE más altas son coincidentes con las zonas de menor pendiente de la cuenca (Fig. 1), con valores que alcanzan los $1110 \mu \mathrm{S} / \mathrm{cm}$.

\section{Agua superficial}

$\underline{\text { Laguna La Brava }}$

La composición química del agua de la laguna es de tipo bicarbonatada sódica presentando gran homogeneidad tanto arealmente como en profundidad (Fig. 3 B). Las muestras se ubican en el extremo sódico del triángulo catiónico del diagrama de Piper, existiendo algunas muestras donde el magnesio también es significativo. En cuanto a los aniones se observa el predominio de los bicarbonatos, aunque existen muestras en donde los cloruros son representativos.

El valor de $\mathrm{pH}$ fluctúa entre 8.50 y 9.39, con un promedio general de 9.17. Se observa una disminución de $\mathrm{pH}$ en profundidad, con una variación en los valores medios que van desde 9.27 a los $0.5 \mathrm{~m}$ hasta 8.58 a los $4 \mathrm{~m}$. La CE varía entre 565 y $658 \mu \mathrm{S} / \mathrm{cm}$, con un promedio

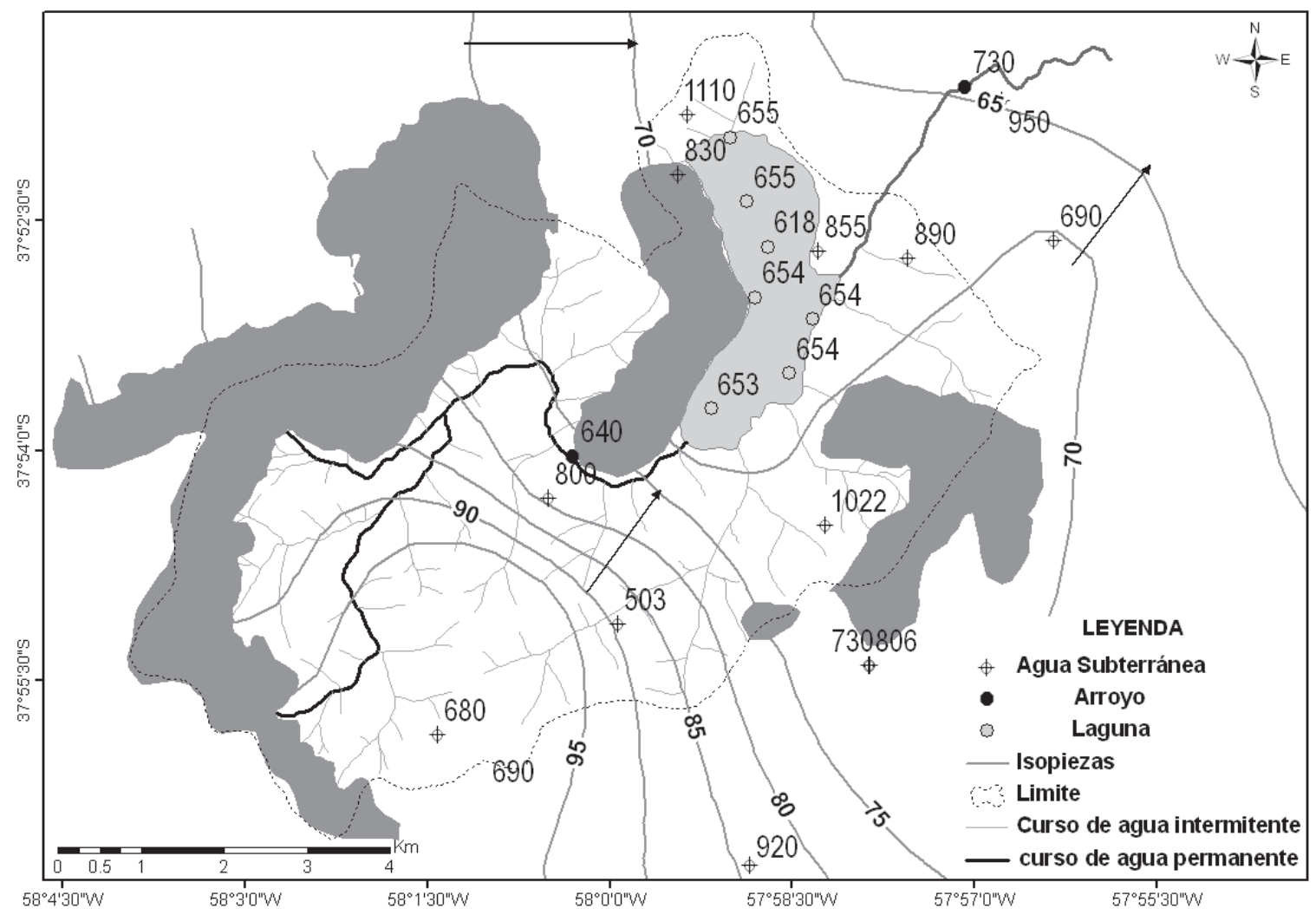

Figura 4. Mapa piezométrico y conductividad eléctrica del agua. Piezometric map and water electrical conductivity. 

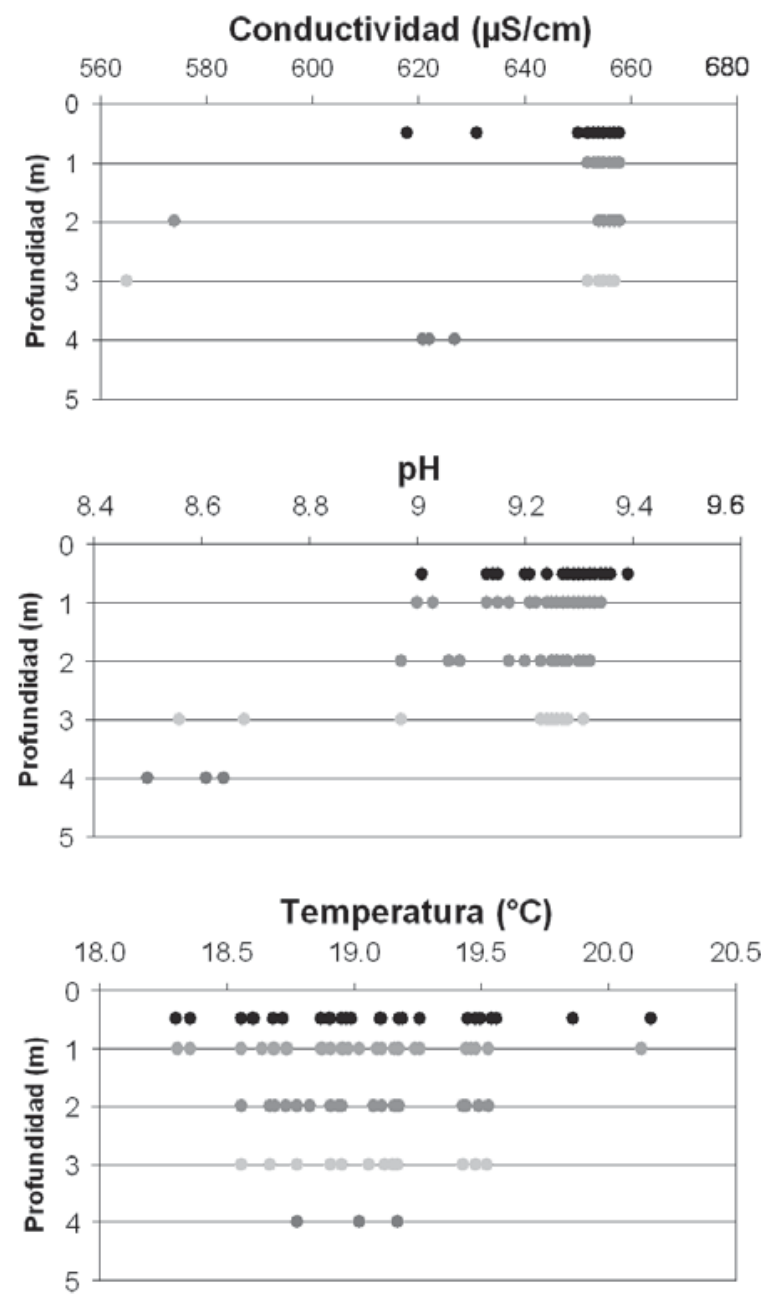

Figura 5. Conductividad eléctrica, $\mathrm{pH}$ y temperatura del agua en función de la profundidad. Electrical conductivity, $p H$, and water temperature variation according to depth.

de $651 \mu \mathrm{S} / \mathrm{cm}$. En la figura 5 se visualizan dos valores diferentes en la $\mathrm{CE}$ a los 2 y 3 metros de profundidad (574 y $565 \mu \mathrm{S} / \mathrm{cm}$ respectivamente). Estas muestras se localizan próximas al faldeo E de la Sierra La Brava, coincidiendo espacialmente con el fallamiento definido para dicha sierra (Mauriño et al. 1981). La temperatura no mostró cambios significativos en profundidad variando entre 18.0 y $20.2^{\circ} \mathrm{C}$ con un valor medio de $19.0^{\circ} \mathrm{C}$. La Tabla 2 muestra los parámetros estadísticos de las variables fisicoquímicas medidas in situ a distintas profundidades.

La distribución espacial de la CE demuestra el predominio de valores superiores a los
Tabla 2. Rango, valor medio y desviación estándar del pH, la conductividad eléctrica (CE) y la temperatura del agua del humedal La Brava a diferentes profundidades. Range, mean and standard deviation of the $\mathrm{pH}$, the electrical conductivity $(C E)$ and the temperature of the water in La Brava wetland at different depths.

\begin{tabular}{clcccc}
\hline $\begin{array}{c}\text { Profundidad } \\
(\mathrm{m})\end{array}$ & Variable & Mínimo & Máximo & Media & DE \\
\hline \multirow{3}{*}{0.5} & $\mathrm{pH}$ & 9.01 & 9.39 & 9.27 & 0.08 \\
& $\mathrm{CE}(\mu \mathrm{S} / \mathrm{cm})$ & 618 & 658 & 652.6 & 8.46 \\
& Temperatura $\left({ }^{\circ} \mathrm{C}\right)$ & 18.3 & 20.17 & 19.07 & 0.45 \\
\hline \multirow{2}{*}{1} & $\mathrm{pH}$ & 9 & 9.34 & 9.25 & 0.09 \\
& $\mathrm{CE}(\mu \mathrm{S} / \mathrm{cm})$ & 652 & 658 & 655 & 1.56 \\
& $\mathrm{Temperatura}\left({ }^{\circ} \mathrm{C}\right)$ & 18.31 & 20.13 & 19.02 & 0.39 \\
\hline \multirow{2}{*}{2} & $\mathrm{pH}$ & 8.97 & 9.32 & 9.23 & 0.1 \\
& $\mathrm{CE}(\mu \mathrm{S} / \mathrm{cm})$ & 574 & 658 & 651.1 & 18.71 \\
& $\mathrm{Temperatura}\left({ }^{\circ} \mathrm{C}\right)$ & 18.56 & 19.53 & 19.02 & 0.3 \\
\hline \multirow{3}{*}{3} & $\mathrm{pH}$ & 8.56 & 9.31 & 9.16 & 0.24 \\
& $\mathrm{CE}(\mu \mathrm{S} / \mathrm{cm})$ & 565 & 657 & 648.7 & 24.13 \\
& $\mathrm{Temperatura}\left({ }^{\circ} \mathrm{C}\right)$ & 18.56 & 19.52 & 19.08 & 0.31 \\
\hline \multirow{4}{*}{4} & $\mathrm{pH}$ & 8.5 & 8.64 & 8.58 & 0.07 \\
& $\mathrm{CE}(\mu \mathrm{S} / \mathrm{cm})$ & 621 & 627 & 623.3 & 3.21 \\
& $\mathrm{Temperatura}\left({ }^{\circ} \mathrm{C}\right)$ & 18.78 & 19.17 & 18.99 & 0.2 \\
\hline
\end{tabular}

$655 \mu \mathrm{S} / \mathrm{cm}$ (Fig. 6 A), se puede observar una zona de menor conductividad localizada en el sector oeste de la laguna en contacto con el faldeo de la Sierra La Brava, con valores promedios de $569 \mu \mathrm{S} / \mathrm{cm}$. Con respecto al $\mathrm{pH}$ y a la temperatura, los valores medidos resultaron ser muy homogéneos existiendo cierta zonación causada posiblemente por la diferencia horaria en la toma de las mediciones (Fig.6B y C). En la elaboración de los mapas de distribución espacial de $\mathrm{pH}, \mathrm{CE}$ y temperatura se consideraron los datos obtenidos a $2 \mathrm{~m}$ de profundidad, dado que en ésta se evidencian variaciones no detectadas a otras profundidades.

\section{Arroyos}

Se tomaron un total de 10 muestras en los arroyos SEP y STJ. La composición iónica de estos cursos de agua mostró un buen agrupamiento en los cationes siendo siempre aguas de tipo sódicas (Fig. 3 C). En cuanto a los aniones se observó mayor dispersión en el SEP, siendo en algún ca- 


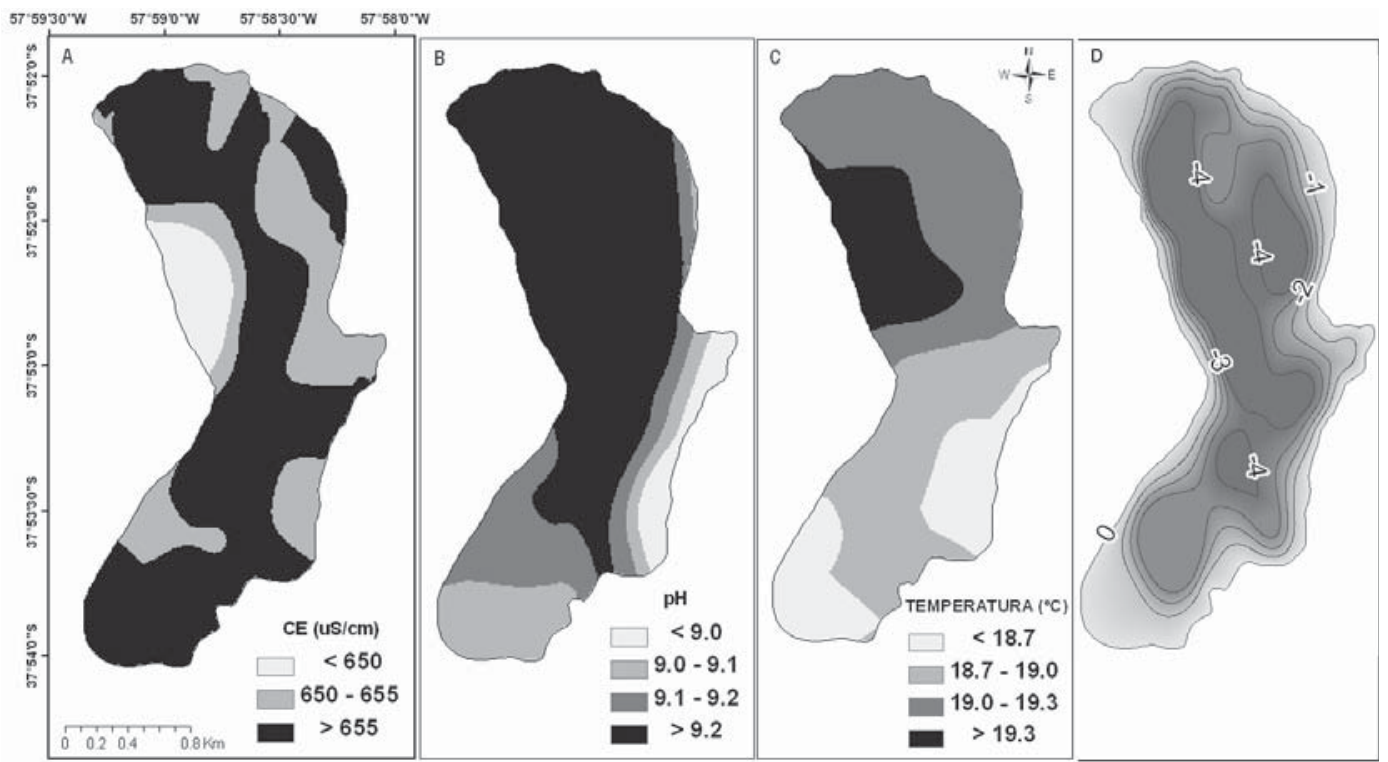

Figura 6. Mapa de distribución de A) Conductividad eléctrica, B) pH y C) Temperatura del agua de La Brava a 2 m de profundidad; D) Mapa batimétrico. Distribution map of A) electrical conductivity, B) $\mathrm{pH}$, and C) Water temperature in La Brava wetland at a depthe of $2 \mathrm{~m}$; D) Bathymetric map.

so de tipo clorurada bicarbonatada; para STJ los bicarbonatos predominaron en todos los casos.

La CE en STJ oscila entre 457 y $801 \mu \mathrm{S} / \mathrm{cm}$ con un valor promedio de $629 \mu \mathrm{S} / \mathrm{cm}$, coincidiendo los menores valores con épocas más lluviosas. En SEP los valores medidos varían entre 640 y $684 \mu \mathrm{S} / \mathrm{cm}$ con un valor medio de $658 \mu \mathrm{S} / \mathrm{cm}$. El $\mathrm{pH}$ y temperatura promedio del agua en SEP y STJ es de 7.6 y $19.1{ }^{\circ} \mathrm{C}$, y de 7.85 y $20.0^{\circ} \mathrm{C}$, respectivamente.

El estudio de mezcla de aguas para identificar la proporción relativa de las principales fuentes de recargar al humedal (agua subterránea y agua de lluvia), utilizando el ión cloruro, muestra que el $61 \%$ del agua del mismo corresponde a agua de lluvia. El análisis de proporción de mezcla para SEP demuestra el predominio del flujo base en el arroyo, por lo tanto éste, no fue considerado como una fuente distinta de recarga al humedal, sino que fue incluido como un componente encauzado de agua subterránea.

\section{Caracterización isotópica}

Los resultados del análisis isotópico de las diferentes muestras analizadas y su relación con la línea meteórica mundial (LMM) se ilustran en la figura 7. En este gráfico puede observarse un agrupamiento en la mayoría de las muestras de agua subterránea, lo que sugiere un sistema bien mezclado a partir del agua de lluvia y confirma el origen de la recarga al acuífero a partir de la precipitación. Dos muestras de agua subterránea presentan una composición isotópica diferente, ambas relacionan el flujo subterráneo con la La-

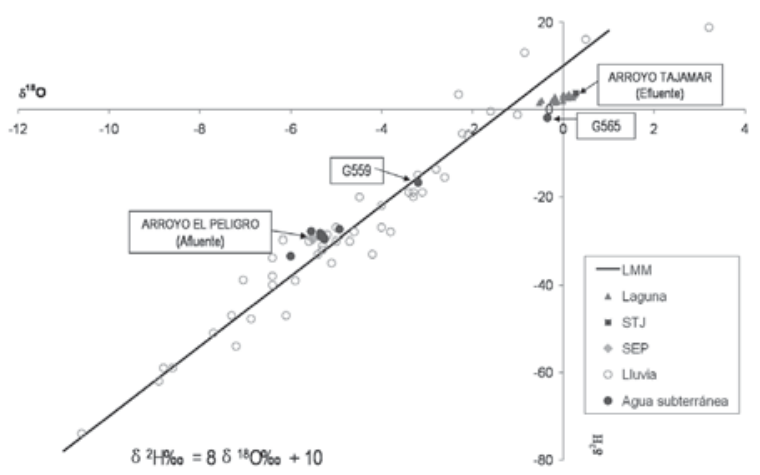

Figura 7. Contenido isotópico $\left(\delta^{18} \mathrm{O}\right.$ y $\left.\delta^{2} \mathrm{H}\right)$ en el agua subterránea, arroyos, laguna y agua de lluvia. Isotopic content $\left(\delta^{18} O\right.$ y $\left.\delta^{2} H\right)$ in the groundwater, streams, wetland, and rainfall. 
guna La Brava y demuestran la influencia que tiene ésta sobre el acuífero en la zona noroeste. Una de las muestras, la más cercana a la laguna (G565), presenta una marca isotópica muy parecida al agua del humedal. Por su parte, la marca isotópica de la muestra G559 evidencia una mezcla entre el agua de la laguna y el acuífero. Lo anterior corrobora la recarga al acuífero desde la laguna, y su comportamiento efluente-influente.

Las muestras de la laguna indican aguas enriquecidas con un valor promedio de -0.05 en $\delta^{18} \mathrm{O}$ y de 2.57 en $\delta^{2} \mathrm{H}$. Las muestras obtenidas en los arroyos permiten definir para SEP una similitud al agua subterránea, mientras que por su parte, STJ presenta un contenido isotópico similar al del agua de la laguna con un mayor proceso de evaporación.

\section{DISCUSIÓN}

Los datos hidroquímicos e isotópicos permitieron validar el modelo hidrogeológico conceptual de la laguna La Brava propuesto por Kruse (1987), confirmando su carácter efluente-influente con el acuífero. De igual manera, estos datos definieron la relación que este humedal tiene con SEP y STJ. El primero aportando agua subterránea encauzada en la parte alta de la cuenca a la laguna y el segundo sirviendo como salida de agua superficial de la misma.

El análisis iónico establece la similitud del tipo de agua dentro de la cuenca, clasificándola como bicarbonatada sódica. Por el contrario son marcadas las diferencias en la CE de las distintas fuentes. Para la laguna se detectaron los valores más bajos, similares a los registrados en el acuífero en zonas definidas como de recarga. Estos valores de CE no se ajustarían al modelo hidrogeológico conceptual definido por Kruse (1987), ya que al ser la laguna recargada básicamente por agua subterránea y SEP, debería poseer una salinidad al menos igual a sus fuentes de recarga y, por lo tanto, mayor a la registrada en los muestreos. Esto hace pensar en la existencia de otra fuente de recarga con menor contenido de sales. El análisis de mezcla de aguas establece que el agua de la laguna corresponde principalmente a precipitación. Esto explicaría las bajas
CE encontradas en el agua de este humedal pese al carácter evaporado de las mismas, evidenciado por su contenido isotópico. El análisis de la $\mathrm{CE}$ a diferentes profundidades sugiere una zona de recarga preferencial en el contacto de la laguna con el faldeo oeste de la Sierra La Brava coincidiendo con el lineamiento definido por Mauriño et al. (1981). En este sector la CE disminuye con la profundidad, sugiriendo un aporte desde el sistema de fisuras presente en las ortocuarcitas. La homogeneidad térmica del humedal descarta la posibilidad de que los valores bajos de CE obedezcan a una disminución en la temperatura, dada la correlación positiva existente entre estos dos parámetros (Hem, 1992).Todo lo anterior indica que los volúmenes de agua que circulan a través de la laguna son altos, evitando que la evaporación cause el incremento de la salinidad/CE.

Un aspecto a tener en cuenta es que no se registraron lluvias intensas en las semanas previas a la toma de muestras. Esto descarta un posible efecto de dilución de las aguas del humedal para este muestreo como consecuencia de precipitaciones directas. Esto sugiere un aporte diferido de precipitaciones, en forma de escurrimiento subsuperficial desde las distintas márgenes de la laguna, especialmente desde el faldeo de la sierra La Brava. Lo anterior coincide con el modelo hidrogeológico propuesto por González (2005) para la región serrana en el esquema de regiones hidrogeológicas de la Provincia de Buenos Aires, donde se plantea una recarga por concentración del escurrimiento superficial desde el frente montañoso, produciéndose infiltración preferencial en coincidencia con la existencia de materiales permeables y fisuras.

El conocimiento de la relación de esta laguna con el acuífero resulta de suma importancia, si se considera que este humedal contribuye al mantenimiento de los niveles freáticos de su zona de influencia. Además, influye sobre la calidad química del agua subterránea filtrando sedimentos y contaminantes potenciales (a través de su vegetación litoral) proveniente de su cuenca de drenaje, y por lo tanto, actuando sobre la calidad del agua para fines domésticos y agrícolas. La presencia de este tipo de humedales puede producir cambios significativos en las características químicas 
del acuífero al ser fuente de recarga de aguas con pH más altos y conductividades más bajas.

Los resultados de este trabajo dejan de manifiesto la importancia hidrogeológica que este humedal tiene para el acuífero. Esto debe ser considerado en las políticas de gestión que se tienen para este cuerpo de agua, las cuales hasta el momento sólo consideran los servicios ecosistémicos relacionados con la provisión de oportunidades de recreación y el soporte de la biodiversidad. El modelo hidrogeológico ajustado obliga a pensar en la Cuenca de la Laguna La Brava como una zona de alta vulnerabilidad para el acuífero y como fuente de recarga al mismo. Esta característica debe ser incluida en los planes de ordenamiento del territorio como factor clave a la hora de llevar a cabo los diferentes emprendimientos urbanísticos que alrededor de este cuerpo de agua se vienen desarrollando. Implementar acciones que ayuden a mejorar la retención de agua en las laderas de la sierra y posterior infiltración contribuiría a mantener la calidad y cantidad del agua en el humedal y subsecuentemente del agua subterránea en la zona de influencia del mismo.

\section{CONCLUSIONES}

Toda la información generada en este trabajo, a partir de la interpretación de datos hidroquímicos e isotópicos, permite validar y ajustar el modelo hidrogeológico de este humedal especialmente en lo referente a las fuentes de entrada al sistema. Las principales fuentes de recarga a la laguna son el agua de lluvia (61 \%) y el agua subterránea (39\%). El agua meteórica recarga al humedal de manera directa e indirectamente en forma de escurrimiento superficial y subsuperficial desde el frente montañoso, en forma preferencial a través de sistema de fisuras presente en las ortocuarcitas que conforman las sierras aledañas. Por su parte, el agua subterránea recarga al humedal también de forma directa y/o en forma encauzada a través del arroyo SEP. La descarga del humedal se realiza a través del arroyo STJ y por la infiltración de agua al acuífero especialmente en la zona norte.

Teniendo en cuenta las relaciones de la laguna La Brava con el acuífero, este cuerpo de agua de- be ser considerado en el modelo hidrológico regional como una zona de recarga al sistema subterráneo y como una zona de alta vulnerabilidad del mismo. Además, debe tenerse en cuenta su contribución en el mantenimiento de los niveles freáticos y en la calidad del agua tanto en forma subterránea como superficial siendo la fuente de abastecimiento del arroyo STJ.

Las marcas isotópicas de cada componente del sistema hídrico en la Cuenca de la Laguna La Brava permitieron corroborar claramente las interacciones entre las distintas fuentes de agua en la misma. Los parámetros que más información proporcionaron para el ajuste y validación de este modelo fueron, la CE en la identificación de zonas de recarga preferenciales, los cloruros utilizados para calcular el porcentaje de mezcla entre las fuentes principales de recarga y el contenido isotópico para establecer las interacciones entre el agua superficial, subterránea y meteórica. Todos estos parámetros sugieren altas tasas de renovación de agua en la laguna.

\section{AGRADECIMIENTOS}

Los autores agradecen al Téc. Hidrógrafo A. Ferrante por su colaboración en los muestreos y al Téc. Químico G. Bernava por los análisis químicos realizados. Estos estudios fueron financiados por el Organismo Internacional de Energía Atómica (OIEA) por el CRP "Isotopic techniques for assessment of hydrological processes in wetlands", la Agencia Nacional de Promoción Científica y Tecnológica (ANPCyT) PICT 0700390 y la Universidad Nacional de Mar del Plata.

\section{REFERENCIAS}

APHA. 1992. Standard methods for the examination of water and wastewater. $18^{\text {th }}$ ed. American Public Health Association, Washington, DC. 1100 pp.

CALMBACH \& WATERLOO HYDROGEOLOGIC. 2003. Water Quality Data Analysis, Plotting, and Modeling; Aquachem User's Manual v.4.0., Co-Developed by Lukas Calmbach and Waterloo Hydrogeologic, Inc., United States of America, 276 pp. 
CLARK, I. \& P. FRITZ. 1997. Environmental Isotopes in Hydrogeology. Lewis Publisher, New York, $328 \mathrm{pp}$.

CUSTODIO, E. \& M. LLAMAS. 1976. Hidrología Subterránea. 2 Tomos, Ed. Omega, Barcelona, $2359 \mathrm{pp}$.

DE GROOT, R., M. STUIP, M. FINLAYSON \& N. DAVIDSON. 2006. Valuing wetlands: Guidance for valuing the benefits derived from wetland ecosystem services. Ramsar Technical Report No. 3, CBD Technical Series No. 27, Gland, Switzerland, $54 \mathrm{pp}$.

ESRI, Environment System Research Institute. 2007. Home page: http://www.esri.com.

FONTES, J. C. H. 1980. Environmental Isotopes in groundwater hydrology. In: Handbook of Environmental Isotopes Geochemistry. Fritz, P., Fontes, J. C. H (eds.): 75-140. Vol. I. The terrestrial Environment, Elsevier, Amsterdam.

GONFIANTINI, R. 1978. Standards for stable isotope measurements in natural compounds. Nature, 271: 534-536.

GONZÁLEZ, N. 2005. Los ambientes hidrogeológicos de la Provincia de Buenos Aires. En: Geología y Recursos Minerales de la Provincia de Buenos Aires. R. E de Barrio, R. O. Etcheverry, M. F. Caballé y E. Llambías (eds.): 359-374. La Plata, Relatorio del XVI Congreso Geológico Argentino.

GROSMAN, F. \& P. SANZANO. 1999. Estudio ictiologico Laguna La Brava, Partido de Balcarce, Provincia de Buenos Aires. Informe Final. Facultad de Ciencias Veterinarias. Universidad Nacional del Centro, $26 \mathrm{pp}$.

HEM, J. D. 1992. Study and Interpretation of the Chemical Characteristics of Natural Waters. U.S.G.S. Water-Supply Paper 2254, fourth printing, 263 pp.

KRUSE, E. 1987. El agua subterránea en el régimen hidrológico de Laguna la Brava. Informe 40, Comisión de Investigaciones Científicas de la Provincia de Buenos Aires, 18 pp.

LIRIO, J. M., H. J NÚÑEZ, M. A. CHAPARRO, A. M. SINITO, A. IRURZUN \& C. S GOGORZA. 2007. Laguna La Brava, Provincia de Buenos Aires. Relaciones paleoclimáticas con Patagonia y Antártida. VI Simposio Argentino y III Latinoamericano sobre Investigaciones Antárticas. Buenos
Aires, Argentina, 1-5 pp.

MA, Millennium Ecosystem Assessment. 2005. Ecosystems and Human Well-Being: Wetlands and Water Synthesis. Millennium Ecosystem Assessment report to the Ramsar Convention. World Resources Institute, Washington, DC, $80 \mathrm{pp}$.

MARTÍNEZ, D., E. M. BOCANEGRA \& M. MANZANO. 2000. La modelación Hidrogeoquímica como herramienta en estudios Hidrogeológicos. Boletín Geológico y Minero. Vol. 111: 83-98.

MAURIÑO, V., O. VARDE \& R. SCANAVINO. 1981. Investigaciones geológicas y geotécnicas realizadas para el proyecto de una central hidroeléctrica de acumulación por bombeo. VIII Congreso Geológico Argentino, Actas II, 45-66. San Luis.

MAZOR, E. 1991. Chemical and Isotopic Groundwater Hydrology. Marcel Dekker, New York, 413 pp.

QUIROZ LONDOÑO, O. M., D. E. MARTÍNEZ, C. DAPEÑA, \& H MASSONE. 2008. Hydrogeochemistry and isotope analyses used to determine groundwater recharge and flow in low-gradient catchments of the province of Buenos Aires, Argentina. Hydrogeology Journal, 16: 1113-1127.

ROMANELLI, A., M. L. LIMA, H. MASSONE \& A. ESCALANTE. 2009. Caracterización hidroquímica subterránea y su relación con la cobertura del suelo en el Corredor Mar del Plata-Balcarce, Prov. de Buenos Aires. XXII Congreso Nacional del Agua (CONAGUA), Trelew, Argentina. 60-71 pp.

SALA, J. M. 1975. Recursos Hídricos (especial mención de las aguas subterráneas). Relatorio Geología de la Provincia de Buenos Aires, IV Congreso Geológico Argentino, Buenos Aires, Setiembre 1975, $169 \mathrm{pp}$.

SOPHOCLEUS, M. 2002. Interaction between Groundwater and Surface Water: The State of the Science. Hydrogeology Journal, 10: 52-67.

TAPIA, A. 1937. Una investigación geológica en la Brava. Revista Geográfica Americana. 167-176.

THORNTHWAITE, C. W. 1948. An approach towards a rational classification of climate. Geographycal review, 38: 55-94.

WINTER, T. C. 1999. Relation of streams, lakes, and wetlands to groundwater flow systems. Hydrogeol. J., 7: 28-45. 


\section{INSTRUCCIONES PARA LOS AUTORES}

\section{Ámbito de publicación de Limnetica}

Limnetica publica artículos originales de investigación sobre la ecología de las aguas continentales. El ámbito de publicación de Limnetica incluye la ecología de ríos, lagos, embalses, lagunas costeras, zonas húmedas, biogeoquímica, paleolimnología, desarrollo de metodologías, taxonomía, biogeografía y todos los aspectos de la ecología acuática continental teórica y aplicada como gestión y conservación, evaluación de impactos, ecotoxicología y contaminación. Por este motivo Limnetica aceptará para su publicación artículos científicos presentando avances del estado del conocimiento, de desarrollo tecnológico así como los que resulten de aplicaciones prácticas novedosas en las especialidades de interés de la revista.

\section{Presentación de manuscritos}

Los autores interesados deberán enviar un manuscrito vía e-mail al Editor de la revista (jarmengol@ub.edu). También se podrá enviar por correo ordinario un original más dos copias en papel y una en soporte magnético (CD, DVD o similares) al Editor de la revista. La copia en soporte informático estandarizado incluirá un fichero único con el texto, las tablas y las figuras según las presentes normas de publicación. Este fichero estará en un editor de textos compatible con un ordenador tipo PC (preferentemente Word, o programa compatible).

Tanto los originales impresos como las copias en soporte magnético se realizarán en hojas tipo A-4 y estarán escritos a doble espacio. Los artículos no sobrepasarán las 6000 palabras en el texto ni 25 hojas impresas (incluidas figuras y tablas) y podrán estar escritos en castellano o en inglés. Excepcionalmente, y previa consulta con el Editor, se podrán presentar manuscritos de mayor longitud que versen sobre revisiones generales, sistemática de grupos taxonómicos amplios o estudios regionales comparativos de un mismo tipo de ecosistemas acuáticos. No se aceptaran aquellos trabajos que no cumplan las presentes instrucciones de publicación.

El Comité Editorial de Limnetica decidirá sobre la publicación o no de los trabajos recibidos, e informará de ello a los autores. Los autores recibirán antes de su publicación una copia de las primeras pruebas de imprenta de su trabajo para su corrección. Una vez publicado el trabajo, el autor responsable de la publicación recibirá una copia en formato pdf.

\section{Estructura del manuscrito}

Todas las palabras en MAYÚSCULAS se acentuarán tanto en el TÍTULO como en los apartados (INTRODUCCIÓN, etc.).

La primera página del manuscrito ha de contener los siguientes apartados:

- Título en mayúsculas.

- Relación de autores con indicación del autor responsable a efectos de la correspondencia. Se deberá indicar expresamente la dirección e-mail de dicho autor.

- Dirección postal completa de los autores.

- Título abreviado.

La segunda página incluirá el Resumen en castellano, palabras clave, el Abstract en inglés y keywords. Tanto el Resumen como el Abstract no deberán sobrepasar las 400 palabras y deberán incluir el título del trabajo en el idioma correspondiente.

Las siguientes páginas se ordenarán en apartados que se estructurarán al estilo científico. Los apartados y párrafos del texto comenzarán sin sangrado. Se acentuarán las mayúsculas en todos los casos.

Los apartados se escribirán sin numerar y se escalarán según el siguiente formato:

Apartado primario.- Mayúsculas y en negrita (INTRODUCCIÓN).

Apartado secundario.- Minúsculas y en negrita.

Apartado terciario.- Itálica.

Apartado de cuarto nivel.- Letra normal subrayada.

Siguientes niveles.- numéricos (1), (1.1), (1.1.1), etc

Las Tablas constituyen una de las partes más costosas en tiempo y presupuesto por lo que se ruega se preparen procurando ocupar el mínimo espacio posible. Las tablas pueden tener la anchura de una columna $(8 \mathrm{~cm}) \mathrm{o}$ dos columnas $(16 \mathrm{~cm})$ y su longitud no puede exceder de $25 \mathrm{~cm}$. Se incluirán al final del manuscrito y tendrán numeración arábiga. En el texto siempre se citarán de forma completa (p.e. Según se puede ver en la Tabla 6... etc. o, Los datos (Tabla 6) indican que... etc.) y nunca en forma abreviada -Tab. 6 o tab. 6. Las leyendas de las tablas se presentarán en castellano e inglés y se incluirán en el mismo apartado que el texto de las figuras. No deberán usarse líneas verticales y los encabezamientos de las columnas deberán ser breves. Se prestará particular atención en no publicar tablas que dupliquen información que ya está en forma de figuras.

Las figuras tendrán numeración arábiga con el texto explicativo en el pie. El texto incluirá la versión en castellano y en inglés. Las figuras pueden ir a tres tipos de caja $8,12.5$ y $16 \mathrm{~cm}$. Los autores procurarán que los originales tengan el tamaño de letra y el grueso de línea necesario para que al reducirse puedan ser interpretables y legibles. No se aceptarán figuras que no cumplan este requisito.

Los pies de figura, junto con los encabezamientos de las tablas, estarán en una página aparte situada inmediatamente después de la bibliografía y antes de las tablas y figuras.

Las citas de las figuras en el texto se harán de forma completa y en minúscula cuando se inserte dentro del texto de un párrafo (p.e. En la figura 1 se indica la situación de los puntos de muestreo). Por el contrario, se citará de forma abreviada y en mayúscula cuando esté entre paréntesis y no relacionada directamente con el texto del párrafo [p.e. Las muestras se han recogido en cinco estaciones distribuidas a lo largo del río (Fig. 1) y con una periodicidad mensual]. Las figuras y fotografías en color sólo se aceptarán de forma excepcional y previa consulta con el Editor.

Las unidades se expresaran preferiblemente en el Sistema Internacional (S.I.) con los símbolos en forma abreviada cuando vayan precedidos de una expresión numérica. Cuando se exprese un valor como combinación de dos unidades éstas se indicarán con el signo aritmético correspondiente p.e. m/s, $\mathrm{mol} / \mathrm{m}^{3}$, ind $/ 1$, pero para más de dos unidades se usarán exponentes, p.e. $\mathrm{m} / \mathrm{s}$, $\mathrm{mol} / \mathrm{m}^{3}$ ind $/ \mathrm{l}$, pero para más de dos unidades se usarán exponentes, p.e. $\mathrm{mgC}$ $\mathrm{m}^{-2} \mathrm{~h}^{-1} \mu \mathrm{mol} \mathrm{m} \mathrm{s}^{-2}$.

Las cantidades con decimales se expresarán con un punto (4.36), los miles con cuatro números sin ninguna separación o símbolo (4392) y para valores iguales o superiores a las decenas de mil se intercalarán blancos separando los miles (13 723 o 132 437). Siempre que sea posible se indicarán los números con notación exponencial decimal con el mínimo posible de decimales (13.7 . $\left.10^{3}, 13.2 \cdot 10^{4}\right)$.

La BIBLIOGRAFÍA se ordenará al final del texto, alfabéticamente y cronológicamente para cada autor, según las pautas siguientes:

- Revistas:

RUEDA, F. J., E. MORENO-OSTOS \& J. ARMENGOL. 2006. The residence time of river water in reservoirs. Ecological Modelling, 191: 260275.

GRAÇA M. A. S. \& C. CANHOTO. Leaf litter processing in low order streams. Limnetica, 25(1-2): 1-10.

RECHE, I., E. PULIDO-VILLENA, R. MORALES-BAQUERO \& E. O. CASAMAYOR. 2005. Does ecosystem size determine aquatic bacterial richness? Ecology, 86: 1715-1722.

- Libro:

KALFF, J. 2002. Limnology. Prentice Hall. NJ. USA. 592 pp.

- Capítulo de libro:

IMBODEN, D. M. 1998. The influence of Biogeochemical Processes on the Physics of Lakes. In: Physical Processes in Lakes and Oceans. J. Iberger (ed.): 591-612. American Geophysical Union. Washington. USA.

- Congresos:

GEORGE, D. G. 2006. Using airborne remote sensing to study the mixing characteristics of lakes ans reservoirs.10th European Workshop on Physical Processes in Natural Waters. June 26-28, 2006. Granada, Spain: 2001-207.

- Informes:

DOLZ, J. \& E. VELASCO. 1990. Analisis cualitativo de la hidrología superficial de las cuencas vertientes a la marisma del Parque Nacional de Doñana (Informe Técnico). Universidad Politécnica de Cataluña. 152 pp.

- Tesis y Maestrias:

MORENO-OSTOS, E. 2004. Spatial dynamics of phytoplankton in El Gergal reservoir (Seville, Spain). Ph.D. Thesis. University of Granada. 354 pp.

THOMPSON, K. L. 2000. Winter mixing dynamics and deep mixing in Lake Tahoe. Master's Thesis, University of California, Davis. 125 pp.

En el manuscrito se listarán únicamente los trabajos citados en el texto; en éste, las referencias se harán en minúsculas (Kalff, 2002; Dolz \& Velasco, 1991; Rueda et al., 2006). En ningún caso se aceptarán como referencias trabajos no publicados (p.e. en preparación) o aún no aceptados (p.e. enviado). Sí se podrán incluir citas de trabajos aceptados para su publicación (en prensa). Se recuerda la conveniencia de reducir al máximo las referencias bibliográficas de difícil consulta como informes, resúmenes a congresos, etc. 


\section{INSTRUCTIONS FOR AUTHORS}

Scope

Limnetica publishes original research papers on ecology of continental waters. Its scope includes ecology of rivers, lakes, reservoirs, lagoons and wetlands, biogeochemistry, paleolimnology, development of new methods, taxonomy, biogeography, and all aspects of theoretical and applied continental aquatic ecology, like management and conservation, impact assessment, ecotoxicology and pollution. Limnetica will accept for publication scientific papers presenting advances in knowledge or technological development, as well as papers derived from new practical approaches on the topics covered by the journal.

\section{Manuscript presentation}

Manuscripts must be submitted by e-mail to the journal Editor (jarmengol@ub.edu). Manuscripts also can be sent to the Editor by regular mail (original plus two hard copies and one digital copy. The digital copy must include a file with text, tables and figures following the present instructions, made with PC-compatible text-edition software (MSWord, Wordperfect, etc.).

Both hard and digital copies will be typed at double space on A-4 sheets. Papers can be written in Spanish or English, and must not exceed 6000 words of text nor 25 printed pages (figures and tables included). Exceptionally, and after consulting the Editor, longer manuscripts can be published for general reviews, systematics of broad taxonomic groups, or regional comparative studies of one kind of aquatic ecosystems. Papers that do not follow the present instructions will be rejected.

Limnetica's Editorial Board will decide whether to publish or not the received manuscripts, and will tell their decision to the authors. Prior to publication, authors will get galley proofs to be corrected. When the paper has been published, the leading author will get a copy in pdf format.

\section{Manuscript structure}

For manuscripts in Spanish, words in UPPER CASE will be accentuated when convenient, both in the title and section headings (INTRODUCCIÓN, etc.).

The first page must include:

- Title in upper case.

- List of authors detailing the corresponding author, whose e-mail address must be shown.

- Complete postal address of authors.

- Running title.

The second page will include Abstract and key words, both in English and Spanish. Abstracts must start with the title and not exceed 400 words.

Following pages must be structured in sections following the scientific style. Section headings and text will have no left indent. Upper case words in Spanish will be accentuated.

Sections and subsections will not be numbered, and must adjust to the following format:

Main section.- Bold, upper case (INTRODUCTION).

$2^{\text {nd }}$-level section.- Bold, lower case.

$3^{\text {rd }}$-level section.- Italics.

$4^{\text {th }}$-level section.- Plain text, underlined.

Lower-level sections.- They will go numbered (1), (1.1), (1.1.1), etc.

Tables are one of the most costly parts, both in terms of time and money; therefore, they must be drawn as compact as possible. Tables can be 1-column $(8 \mathrm{~cm})$ or 2-column $(16 \mathrm{~cm})$ wide, and their length cannot exceed $25 \mathrm{~cm}$. They will be included at the end of the manuscript and numbered in Arabic numbers In the text they will be written in complete form (e.g., as can be seen in Table $6 \ldots$ or Data (Table 6) show that...), never in abbreviated form (neither Tab. 6 nor tab. 6). Table captions will be written in both English and Spanish, and will be included in the text in the same section than Figure legends. No vertical lines can be drawn in tables, and column headings must be short. No table will be published that shows information presented in figures.

Figures will have Arabic numbers, and legends will go below, both in English and Spanish. Figures can fit three box-sizes: $8 \mathrm{~cm}, 12.5 \mathrm{~cm}$, or $16 \mathrm{~cm}$.
Authors must make sure that font size and line thickness can be easily read after reduction, otherwise figures will be rejected.

Figure legends and table captions will go in a page after Literature Cited and before Tables and Figures.

Figure calls must be made in complete, lower case form when in the text (e.g., Location of sampling sites is shown in figure 1), in abbreviated, upper case when going in a parenthesis and not directly related to the text [e.g., Samples were taken monthly at five sites along the river (Fig. 1)]. The Editor will accept to publish colour figures and photographs only exceptionally and when explicitly requested.

Units must be expressed preferably following the International System (I.S.), with abbreviated symbols when preceded by numeric expressions. Values combining two units must be expressed with the corresponding arithmetic sign, like $\mathrm{m} / \mathrm{s}, \mathrm{mol} / \mathrm{m}^{3}$, ind $/ \mathrm{l}$, but when there are more than two units exponentials must be used, like in $\mathrm{mgC} \mathrm{m}^{-2} \mathrm{~h}^{-1}, \mu \mathrm{mol} \mathrm{m} \mathrm{m}^{-2} \mathrm{~s}^{-1}$.

Decimal numbers will be expressed with a dot (4.36), thousands with 4 digits, with no blank space or symbols (4392), and figures over ten thousand will have blank space markings (13 723 or 132437 ). Whenever possible the scientific notation will be used, with the smallest possible number of decimals $\left(13.7 \cdot 10^{3}, 13.2 \cdot 10^{4}\right)$

BIBLIOGRAPHY will be after the text, in alphabetic order, chronologically for each author, and adhere to the following style:

- Journals:

RUEDA, F. J., E. MORENO-OSTOS \& J. ARMENGOL. 2006. The residence time of river water in reservoirs. Ecological Modelling, 191: 260275 .

GRAÇA M. A. S. \& CRISTINA CANHOTO. Leaf litter processing in low order streams. Limnetica, 25(1-2): 1-10

RECHE, I., E. PULIDO-VILLENA, R. MORALES-BAQUERO \& E. O. CASAMAYOR. 2005. Does ecosystem size determine aquatic bacterial richness? Ecology, 86: 1715-1722.

- Books:

KALFF, J. 2002. Limnology. Prentice Hall. NJ. USA. 592 pp.

- Book chapters:

IMBODEN, D. M. 1998. The influence of Biogeochemical Processes on the Physics of Lakes. In: Physical Processes in Lakes and Oceans. J. Iberger (ed.): 591-612. American Geophysical Union. Washington. USA.

CASTRO, M., J. MARTIN-VIDE \& S. ALONSO. 2005. El clima de España: pasado, presente y escenarios de clima para el siglo XXI. In: Evaluación preliminar de los impactos en España por efecto del Cambio Climático. J. M. Moreno Rodríguez (ed.): 113-146. Ministerio de Medio Ambiente.

- Conferences:

GEORGE, D. G. 2006. Using airborne remote sensing to study the mixing characteristics of lakes ans reservoirs.10th European Workshop on Physical Processes in Natural Waters. June 26-28, 2006. Granada, Spain: 2001-207.

- Reports:

DOLZ, J. \& E. VELASCO. 1990. Análisis cualitativo de la hidrología superficial de las cuencas vertientes a la marisma del Parque Nacional de Doñana (Informe Técnico). Universidad Politécnica de Cataluña. 152 pp.

- $\mathrm{PhD}$ and Master Dissertations:

MORENO-OSTOS, E. 2004. Spatial dynamics of phytoplankton in El Gergal reservoir (Seville, Spain). Ph.D. Thesis. University of Granada. $354 \mathrm{pp}$.

THOMPSON, K. L. 2000. Winter mixing dynamics and deep mixing in Lake Tahoe. Master's Thesis, University of California, Davis. 125 pp.

The Bibliography will only contain papers cited in the text, where they must go in lower case (Margalef, 1975; Wetzel \& Likens, 1991; Riera et al., 1992). In no case will unpublished (e.g., in prep., submitted) papers be cited, unless they are accepted for publication (in press). References to works hard to get (reports, conference abstracts, etc.) must be limited to the minimum possible. 